\title{
Long-Term Clinical Outcomes of Percutaneous Coronary Intervention in Saphenous Vein Grafts in a Low to Middle-Income Country
}

\author{
Ghufran Adnan ${ }^{1}$, Intisar Ahmed ${ }^{1}$, Javed Tai ${ }^{1}$, Maria Ali Khan ${ }^{2}$, Hammad Hasan ${ }^{3}$ \\ 1. Cardiology, Aga Khan University Hospital, Karachi, PAK 2. Biostatistics and Epidemiology, Aga Khan University \\ Hospital, Karachi, PAK 3. Cardiology, Queen Alexandra Hospital, Portsmouth, GBR
}

Corresponding author: Javed Tai, javed.tai@aku.edu

\section{Abstract \\ Background}

Revascularization of saphenous vein grafts (SVGs) is challenging and debated for the last few decades. The percutaneous revascularization of SVGs was reported to have poorer long-term outcomes than native coronary artery revascularization.

\section{Purpose}

We aim to study the peri-procedural complications and long-term outcomes of the percutaneous revascularization of SVGs in a low-middle-income country.

\section{Methods}

In this retrospective study, we included 110 patients who underwent percutaneous revascularization from January 2011 to March 2020 and followed them retrospectively for long-term outcomes and major adverse cardiovascular events.

\section{Results}

The mean age was $71 \pm 9$, and $81 \%$ were male. The most common reason for the presentation was non-ST segment elevation myocardial infarction (NSTEMI) (46\%). The mean follow-up period of the study was $48 \pm 27$ months. The most common comorbidity was hypertension (86\%). A drug-eluting stent (80\%) was placed in most of the patients, followed by a bare-metal stent (BMS) (14\%) and percutaneous balloon angioplasty (POBA) (6\%). We did not find any significant difference in major adverse cardiac events (MACE) $(\mathrm{P}=0.48)$, target vessel revascularization (TVR) $(\mathrm{p}=0.69)$, and target lesion revascularization (TLR) $(\mathrm{p}=0.54)$ with drugeluting stent (DES) as compared to either BMS or POBA. The mean period from coronary artery bypass grafting $(\mathrm{CABG})$ to SVG percutaneous coronary intervention $(\mathrm{PCI})$ was $15 \pm 5.5$ years. Multivariate Cox regression analysis showed that an acute coronary syndrome (ACS) event, stroke, and female sex were independently associated with MACE.

Review began 10/20/2020 Review ended 11/05/2020 Published 11/16/2020

(c) Copyright 2020

Adnan et al. This is an open access article distributed under the terms of the Creative Commons Attribution License CC-BY 4.0., which permits unrestricted use, distribution, and reproduction in any medium, provided the original author and source are credited.

\section{Conclusion}

The long-term outcomes of SVG PCI are not affected by the types of stents. Female gender, ACS, and stroke are the independent predictors of MACE after SVG PCI, and statin therapy has a positive impact on the longterm outcomes of SVG PCI.

Categories: Cardiac/Thoracic/Vascular Surgery

Keywords: percutaneous intervention, low to middle income countries, saphenous vein graft

\section{Introduction}

Cardiovascular diseases are the leading cause of death worldwide, and coronary artery disease accounts for more than $40 \%$ of cardiovascular deaths [1]. Coronary artery bypass grafting (CABG) is a recommended revascularization procedure for complex coronary artery disease associated with better long-term outcomes, especially in patients with left main or multivessel coronary artery disease [2].

Although CABGs overall are reported to have a lower rate of repeat revascularization than percutaneous coronary intervention (PCI), saphenous vein grafts (SVGs) are prone to early atherosclerosis and degeneration, leading to graft occlusion [3] As high as 20\% of the SVGs develop obstructive lesions and occluded in the first 18 months due to accelerated intimal hyperplasia [4-5]. At 10 years, more than $50 \%$ of the SVGs were found to be occluded [6-7]. 
The revascularization of SVGs is challenging and has been a matter of debate for the last few decades. A redo coronary bypass graft is not recommended in the setting of a patent internal mammary graft to the left anterior descending artery, as it is associated with high perioperative complications [8]. The percutaneous revascularization of SVGs was reported to have a lower success rate and poor long-term outcomes as compared to native coronary artery percutaneous revascularization [9-10].

SVG intervention is not similar to that of native coronary artery percutaneous coronary intervention (PCI). Many factors described in the literature make SVG intervention challenging. These may include the arterialization of the vein graft due to high pressure, leading to intimal hyperplasia and atherosclerosis, a thin cap of atherosclerotic plaque, which makes it vulnerable for distal embolization intervention, and a large thrombus burden, especially in acute occlusion [11-12]. All these factors, along with the release of neurohumoral factors, lead to no-reflow, which increases the risk of peri-procedure myocardial infarction and affects short and long-term outcomes [13].

As the prevalence of coronary artery disease increases in low-middle-income countries, it is essential to generate local evidence to increase the effectiveness of coronary artery disease treatment strategies. We aim to study the peri-procedural complications, as well as the long and short-term outcomes of SVG PCI in a low-middle-income country.

\section{Materials And Methods}

We conducted a retrospective study at a major tertiary care hospital in Karachi. We enrolled in a cohort of patients from the last 10 years, i.e., 2010-2019, aged $>18$ years old who underwent PCI of SVG. Data were collected by reviewing the medical records of patients who fulfill inclusion criteria 1) >18 years of age, 2) PCI of the saphenous vein graft with either balloon angioplasty or stent (drug-eluting stent (DES) and baremetal stent (BMS)). We excluded the patients who underwent PCI of native arteries.

We assessed demographic parameters, comorbidities at presentation, family history, cardiovascular risk factors, medications, previous PCI, and ejection fraction from the database. Procedural features were also recorded, i.e., native vessel disease, graft vessel disease, PCI of graft, type of procedure, reference vessel, number of stents, stent length, stent diameter, target vessel revascularization, guide catheter, guidewire, aspiration, thrombus, pre-dilation, post-dilation, and percentage of stenosis. We also followed for postprocedure events.

\section{Operational definition}

Event: Major adverse cardiac event (MACE) defined as cardiac death, myocardial infarction, stroke, and revascularization (including both target vessel revascularization and target lesion revascularization).

Target vessel revascularization (TVR): Defined as repeat percutaneous intervention of previously stented SVG graft.

Target lesion revascularization (TLR): Defined as repeat percutaneous intervention within $5 \mathrm{~mm}$ proximal or distal of previously stented SVG graft.

\section{Statistical analysis}

Data were analyzed using statistical software STATA (version 14.2; StataCorp, College Station, TX). In the descriptive analysis, mean and standard deviation were calculated for quantitative variables and proportions for qualitative variables, including the dependent variable. We did a univariate analysis with Cox proportional hazard regression in which association between independent variables was observed with the outcome. The final multivariable Cox regression model was developed using a stepwise approach through model building, considering p-value $<0.05$ statistically significant. After that, the global test for the proportional hazard assumption was performed. Acute coronary syndrome (ACS) event, stroke, and sex remained in the parsimonious model. Survival analysis was demonstrated by Kaplan Meier curves and compared with the log-rank test.

\section{Results}

Between January 2011 and March 2020, a bypass graft angiogram was performed in 650 patients, out of which 111 patients with PCI of SVG were included. The mean age was $71 \pm 9$, and $81 \%$ were male. The most common reason for the presentation was non-ST segment elevation myocardial infarction (NSTEMI) (46\%). The mean follow-up period of the study was $48 \pm 27$ months. The baseline characteristics of the patient are shown in Table 1 . The most common comorbidity was hypertension (86\%), followed by diabetes (66\%), dyslipidemia (24\%), stroke (3.60\%), and chronic kidney disease (CKD) (15\%). 


\section{Cureus}

\begin{tabular}{|c|c|}
\hline Age & $71 \pm 9$ \\
\hline Gender (male) & $90(81 \%)$ \\
\hline Hypertension & $96(86 \%)$ \\
\hline Diabetes & $73(66 \%)$ \\
\hline Chronic kidney disease & $17(15 \%)$ \\
\hline Dyslipidemia & $27(24 \%)$ \\
\hline Stroke & $4(3.6 \%)$ \\
\hline Left ventricle ejection fraction $<45 \%$ & $57(51 \%)$ \\
\hline Previous PCl & $23(21 \%)$ \\
\hline NSTEMI & $51(46 \%)$ \\
\hline STEMI & $11(10 \%)$ \\
\hline Unstable angina & $23(21 \%)$ \\
\hline Stable angina & $26(23 \%)$ \\
\hline Beta-blocker & $97(87 \%)$ \\
\hline Nitrate & $27(24 \%)$ \\
\hline ACE inhibitors & $35(31 \%)$ \\
\hline Calcium channel blocker & $32(28 \%)$ \\
\hline Statin & 109(98\%) \\
\hline Angiotensin receptor blocker & $24(21 \%)$ \\
\hline
\end{tabular}

\section{TABLE 1: Baseline clinical characteristics of patients}

PCI: percutaneous coronary intervention; NSTEMI: non-ST segment elevation myocardial infarction; STEMI: ST-segment elevation myocardial infarction; ACE: angiotensin-converting enzyme

The patients' angiographic and procedural characteristics of SVG PCI are shown in Table 2.

\begin{tabular}{|l|l|}
\hline Native LM Disease & $38(34 \%)$ \\
\hline Native LAD disease & $108(97 \%)$ \\
Native Ramus disease & $11(10 \%)$ \\
Native LCX disease & $104(94 \%)$ \\
Native RCA disease & $106(96 \%)$ \\
LIMA-LAD disease & $14(13 \%)$ \\
SVG-LAD disease & $15(14 \%)$ \\
SVG-Diagonal disease & $29(26 \%)$ \\
SVG-Ramus disease & $8(7 \%)$ \\
SVG-OM disease & $74(67 \%)$ \\
SVG-RCA disease & $68(61 \%)$ \\
Thrombus & $21(19 \%)$ \\
Aspiration & $24(22 \%)$ \\
Stenosis percentage> $90 \%$ & $84(75 \%)$ \\
\hline
\end{tabular}




\section{Cureus}

\section{Guide catheter}

Left coronary bypass catheter

Judkins right

Amplatz left

Multipurpose catheter

Internal mammary catheter

Guidewire

Balance middleweight (BMW) wire

Whisper

Cougar XT

Miracle

Fielder FC

Sion

Dilation

Pre-dilation

Post-dilation

Maximum post-dilation

Stent size

Stent diameter

Stent length

Stent number

Stent type

Drug-eluting stent (DES)

\section{TABLE 2: Patients' procedural characteristics}

LM: left main; LAD: left anterior descending artery; LCX: left circumflex artery; RCA: right coronary artery; LIMA: left internal mammary artery; SVG: saphenous vein graft; OM: obtuse marginal artery

Most patients in our study who had SVG PCI were also found to have significant ( $>50 \%$ for left main, $>70 \%$ for other vessels) disease in native vessels. The most common disease vessels were SVG to OM and SVG to RCA grafts. We observed that the most common graft in which PCI was performed was SVG to OM (52\%). However, patients with a critical disease in a left internal mammary artery (LIMA) graft were less as compared with an SVG graft, yet adverse events significantly occur more in patients with LIMA graft disease. Multivessel graft PCI was performed in $6 \%$ of patients. Most of the grafts engaged with LCB (47\%) and BMW wires $(77 \%)$ were able to cross the lesion successfully. A drug-eluting stent (80\%) was placed in most of the patients, followed by BMS (14\%) and POBA (6\%). We did not find any significant difference in MACE, TVR, and TLR with DES as compared to either BMS or POBA. Nevertheless, the higher the number of stents in a patient, the more the likelihood of adverse outcomes. The average stent diameter $3.23 \pm 0.5$, and length were 


\section{Cureus}

$22.3 \pm 9$. Direct stenting was done in $41 \%$ of patients. It was observed in patients in whom aspiration thrombectomy was performed either for large thrombus burden or no re-flow associated with adverse outcomes. It noted that patients with a high thrombus burden have adverse outcomes.

The mean period from CABG to SVG PCI was $15 \pm 5.5$ years. However, it is noted that significant adverse events occur after five years of SVG PCI ( $\mathrm{p}=0.08)$. The results of the events are shown in Table 3 .

\begin{tabular}{|l|l|}
\hline MACE & $18(16 \%)$ \\
\hline TVR & $10(9 \%)$ \\
\hline TLR & $5(5 \%)$ \\
\hline Death & $9(8 \%)$ \\
\hline Cardiac death & $3(3 \%)$ \\
\hline
\end{tabular}

\section{TABLE 3: Incidence of events}

TVR: target vessel revascularization; TLR: target lesion revascularization

The incidence of all-cause death was nine (8\%), MACE 18 (16\%), target vessel revascularization 10 (9\%), and target lesion revascularization 5 (5\%). Multivariate Cox regression analysis showed that an ACS event, stroke, and female sex were independently associated with MACE. Kaplan Meier survival (Figure 1) showed decreased survival in females, stroke, and acute coronary syndrome (Table 4).

\section{Kaplan Meier curves for long term outcomes in female and Stroke patients}

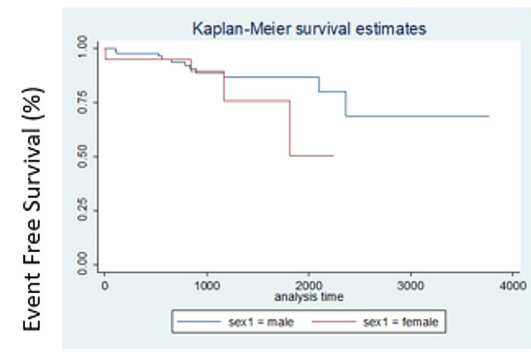

Days since SVG PCI

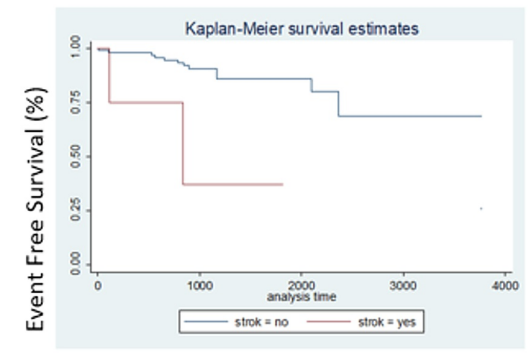

Days since SVG PCI

FIGURE 1: Kaplan Meier survival in females and stroke patients

\begin{tabular}{|c|c|c|c|}
\hline Predictors & HR & p-value & $95 \% \mathrm{Cl}$ \\
\hline Female sex & 3.79 & 0.02 & $1.16-12.4$ \\
\hline Stroke & 9.79 & $<0.001$ & $2.37-40.4$ \\
\hline ACS & 36.8 & $<0.001$ & 7.6-178.2 \\
\hline
\end{tabular}

\section{TABLE 4: Predictors of MACE}

ACS: acute coronary syndrome; MACE: major adverse cardiac event

\section{Discussion}

It is one of the few studies from low and middle-income countries (LMICs) on the long-term outcomes of SVG PCI. Even in the contemporary era of advanced intervention techniques and embolic protection devices, 
studies from high-income and upper-middle-income countries have reported high peri-procedural complications and lower long-term outcomes of SVG PCI. In a meta-analysis by Sidik N et al., the periprocedural complications of SVG-PCI were reported as 16\%, and MACE was as high as $45 \%$ at the 36 months follow-up [14]. According to the ARRIVE (use of aspirin to reduce the risk of initial vascular events in patients at moderate risk of cardiovascular disease) study, patients undergoing SVG PCI with drug-eluted stents had higher two-year mortality, myocardial infarction, and stent thrombosis as compared to those who had native vessel PCI [15]. In another study by Eid-Lidt et al., SVG PCI was associated with high periprocedural complications and lower MACE-free long-term survival [16]. The study reveals that most participants were males, and our study population was relatively older, with a mean age of more than 70 years. In a study by Redfors, the mean age of the participants undergoing saphenous vein graft PCI was 69.3 years, and $84 \%$ were males [17]. Although the male gender is an independent risk factor for coronary artery disease, females were reported to have poor outcomes after coronary intervention due to some reasons [18].

Ahmed et al. studied more than 1,000 patients with SVG grafts and found that female participants were older than males with a mean age of 69 years. Female gender was associated with poor outcomes after the percutaneous intervention [19]. On the other hand, the post-CABG trial showed that the male gender was inclined to have poor results than the female gender [20]. We found that the female gender was independently associated with significant MACE.

More than $85 \%$ of our study population had hypertension, and two-thirds of them had diabetes mellitus. Around $74 \%$ of the patients in our study presented with an ACS, which itself is an independent predictor of poor outcomes irrespective of intervention. Redfors et al. reported hypertension and diabetes in 91\% and $44 \%$ of the study population, respectively, and only $56 \%$ of their patients presented with ACS [17]. In a study from China, $61 \%$ of the patients undergoing graft PCI were hypertensive, and $73 \%$ of the study population presented with ACS [21].

The mean time from CABG to the requirement of SCG PCI in our study population was around 15 years as compared with the other studies [14]. The majority of the patients underwent DES in our study, followed by BMS and POBA, respectively. Although SVG PCI with DES reported being superior to BMS, in terms of longterm outcomes, we did not find a statistically significant difference in the long-term outcomes and MACE among these intervention groups [15,22]. Stent diameter, length of the stent, and pre and post-dilatation were not found to be associated with MACE in our study population. A study by Leborgne supports direct stenting as pre-dilation is related to distal embolization of debris and increases peri-procedure myocardial infarction and increased CK-MB levels [23]. However, a retrospective study did not significantly differ in patients undergoing direct stenting compared to those who underwent pre-dilation in distal embolic protection devices [24].

Enormous thrombus burden and aspiration thrombectomy was associated with adverse outcomes. Enormous thrombus burden, especially in the setting of an ACS, is reported to have poor outcomes in patients undergoing SVG PCI [25]. Aspiration thrombectomy even during native coronary artery revascularization increases the risk of distal embolization and stroke [26]. However, embolic protection devices are recommended during SVG PCI to reduce incidents of distal embolization and no-reflow but those devices were not used in our study population due to limited availability [27].

Other than the female gender, ACS and stroke were independent predictors of MACE in our study population. In a pooled analysis of around 4,000 patients, Coolong et al. found that plaque burden large thrombus and SVG degeneration were the strongest predictors of MACE [28]. An ACS is caused by acute thrombotic occlusion of the SVG graft, which leads to distal embolization and no-reflow. Brodie BR et al. reported poor outcomes of SVG PCI in ST-elevation myocardial infarction due to distal embolization and no-reflow [29].

Our study has some limitations. As this is a retrospective, observational study and patients were not randomized, which may influence the results. The research was done in a single-center, and the sample size was small, which may not represent the whole population in our region. We do not have a comparison group, so it is difficult to establish the association of SVG PCI with mortality. We suggest prospective randomized studies to predict the long-term outcomes of SVG PCI in our region.

\section{Conclusions}

Patients undergoing SVG PCI in our population are relatively older and predominantly male. The long-term outcomes of SVG PCI are not affected by the type of stents. Female gender, ACS, and stroke are independent predictors of MACE after SVG PCI.

\section{Additional Information \\ Disclosures}

Human subjects: Consent was obtained by all participants in this study. Ethical Review Committee, The Aga Khan University Hospital issued approval 2020-3444-10584. Javed Tai: Outcomes of percutaneous 
coronary intervention in tertiary care hospital Thank you for your application for exemption from ethical approval regarding the above-mentioned study. Your study was reviewed and approved as an exemption. Please ensure that the study is performed as per protocol following all AKU standards. Animal subjects: All authors have confirmed that this study did not involve animal subjects or tissue. Conflicts of interest: In compliance with the ICMJE uniform disclosure form, all authors declare the following: Payment/services info: All authors have declared that no financial support was received from any organization for the submitted work. Financial relationships: All authors have declared that they have no financial relationships at present or within the previous three years with any organizations that might have an interest in the submitted work. Other relationships: All authors have declared that there are no other relationships or activities that could appear to have influenced the submitted work.

\section{References}

1. Nowbar AN, Gitto M, Howard JP, Francis DP, Al-Lamee R: Mortality from ischemic heart disease: analysis of data from the World Health Organization and coronary artery disease risk factors from NCD Risk Factor Collaboration. Circulation. 2019, 12:005375. 10.1161/CIRCOUTCOMES.118.005375

2. Serruys PW, Morice MC, Kappetein AP, et al.: Percutaneous coronary intervention versus coronary-artery bypass grafting for severe coronary artery disease. N Engl J Med. 2009, 5:961-972. 10.1056/NEJMoa0804626

3. Lee M, Kong J: Current state of the art in approaches to saphenous vein graft interventions . Interv Cardiol. 2017, 12:85-91. 10.15420/icr.2017:4:2

4. Fitzgibbon GM, Kafka HP, Leach AJ, Keon WJ, Hooper GD, Burton JR: Coronary bypass graft fate and patient outcome: angiographic follow-up of 5,065 grafts related to survival and reoperation in 1,388 patients during 25 years. J Am Coll Cardiol. 1996, 28:616-626. 10.1016/0735-1097(96)00206-9

5. Motwani JG, Topol EJ: Aortocoronary saphenous vein graft disease. Pathogenesis, predisposition, and prevention. Circulation. 1998, 10:916-931. 10.1161/01.CIR.97.9.916

6. Harskamp RE, Lopes RD, Baisden CE, De Winter RJ, Alexander JH: Saphenous vein graft failure after coronary artery bypass surgery: pathophysiology, management, and future directions. Ann Surg. 2013, 257:824-833. 10.1097/SLA.0b013e318288c38d

7. Hess CN, Lopes RD, Gibson CM, et al.: Saphenous vein graft failure after coronary artery bypass surgery. Insights from PREVENT IV. Circulation. 2014, 21:1445-1451. 10.1161/CIRCULATIONAHA.113.008193

8. Cole JH, Jones EL, Craver JM, et al.: Outcomes of repeat revascularization in diabetic patients with prior coronary surgery. J Am Coll Cardiol. 2002, 4:1968-1975. 10.1016/s0735-1097(02)02561-5

9. Grube E, Buellesfeld L: Saphenous vein graft coronary interventions. Eur Cardiol. 2005, 1:42-43. 10.15420/ecr.2005.42

10. Blachutzik F, Achenbach S, Troebs M, Roether J, Nef H, Hamm C, Schlundt C: Angiographic findings and revascularization success in patients with acute myocardial infarction and previous coronary bypass grafting. Am J Cardiol. 2016, 15:473-476. 10.1016/j.amjcard.2016.05.040

11. Hong MK, Mehran R, Dangas G, et al.: Creatine kinase-MB enzyme elevation following successful saphenous vein graft intervention is associated with late mortality. Circulation. 1999, 14:2400-2405. 10.1161/01.CIR.100.24.2400

12. Califf RM, Abdelmeguid AE, Kuntz RE, et al.: Myonecrosis after revascularization procedures. J Am Coll Cardiol. 1998, 31:241-251.

13. Sdringola S, Assali AR, Ghani M, et al.: Risk assessment of slow or no-reflow phenomenon in aortocoronary vein graft percutaneous intervention. Catheter Cardiovasc Interv. 2001, 54:318-324. 10.1002/ccd.1290

14. Sidik N, McCreary K, Abouzaid A, Hanratty C, Walsh S, McEntegart M, Spratt J: TCT-520 long-term outcomes of saphenous vein graft percutaneous coronary intervention. J Am Coll Cardiol. 2018, 72:13.

15. Brilakis ES, Lasala JM, Cox DA, Berger PB, Bowman TS, Starzyk RM, Dawkins KD: Outcomes after implantation of the TAXUS paclitaxel-eluting stent in saphenous vein graft lesions: results from the ARRIVE (TAXUS Peri-Approval Registry: A Multicenter Safety Surveillance) program. JACC Cardiovasc Interv. 2010, 1:742-750. 10.1016/j.jcin.2010.04.012

16. Eid-Lidt G, Gaspar J, Adames AE, Damas de Los SF VR, Ramirez-Gutierrez AE: Long-term outcomes of saphenous vein graft stenting compared with native coronary artery stenting in patients with previous coronary artery bypass graft surgery. Arch Cardiol Mex. 2010, 80:3-9.

17. Redfors B, Généreux P, Witzenbichler B, et al.: Percutaneous coronary intervention of saphenous vein graft . Circulation. 2017, 10:004953. 10.1161/CIRCINTERVENTIONS.117.004953

18. Ang PC, Harper RW, Meredith IT: Angioplasty in women: a re-look into their clinical success and complications. J Am Coll Cardiol. 2000, 35:2.

19. Ahmed JM, Dangas G, Lansky AJ, et al.: Influence of gender on early and one-year clinical outcomes after saphenous vein graft stenting. Am J Cardiol. 2001, 15:401-405. 10.1016/S0002-9149(00)01391-6

20. Domanski MJ, Borkowf CB, Campeau L, et al.: Prognostic factors for atherosclerosis progression in saphenous vein grafts: the postcoronary artery bypass graft (post-CABG) trial. J Am Coll Cardiol. 2000, 15:1877-1883.

21. Liu D, Cui X, Luo X, Sun Z, Xu B, Qiao S, Yuan J: Long-term outcomes of percutaneous coronary intervention in grafts and native vessels in coronary artery bypass grafting patients with diabetes mellitus. J Thorac Dis. 2019, 11:4798-4806. 10.21037/jtd.2019.10.33

22. Mosleh W, Gandhi S, Elsiddig M, Schwalm JD, Farkouh ME: Comparison of drug-eluting stents with baremetal stents for PCI of saphenous vein graft lesions: systematic review and meta-analysis. J Invasive Cardiol. 2016, 1:139-169.

23. Leborgne L, Cheneau E, Pichard A, et al.: Effect of direct stenting on clinical outcome in patients treated with percutaneous coronary intervention on saphenous vein graft. Am Heart J. 2003, 146:501-506. 10.1016/S0002-8703(03)00309-0

24. Okabe T, Lindsay J, Torguson R, et al.: Can direct stenting in selected saphenous vein graft lesions be considered an alternative to percutaneous intervention with a distal protection device?. Catheter Cardiovasc 


\section{Cureus}

Interv. 2008, 15:799-803. 10.1002/ccd.21678

25. Lee MS, Park SI, Kandzari DE, et al.: Saphenous vein graft intervention. JACC Cardiovasc Interv. 2011, 4:831843.

26. Jolly SS, Cairns JA, Yusuf S, et al.: Randomized trial of primary PCI with or without routine manual thrombectomy. N Engl J Med. 2015, 9:1389-1398. 10.1056/NEJMoa1415098

27. Levine GN, Bates ER, Blankenship JC, et al.: 2011 ACCF/AHA/SCAI guideline for percutaneous coronary intervention: executive summary. A report of the American College of Cardiology Foundation/American Heart Association Task Force on Practice Guidelines and the Society for Cardiovascular Angiography and Interventions. Catheter Cardiovasc Interv. 2012, 15:453-495. 10.1161/CIR.0b013e31823a5596

28. Coolong A, Baim DS, Kuntz RE, et al.: Saphenous vein graft stenting and major adverse cardiac events. A predictive model derived from a pooled analysis of 3958 patients. Circulation. 2008, 12:790-797. 10.1161/CIRCULATIONAHA.106.651232

29. Brodie BR, VerSteeg DS, Brodie MM, et al.: Poor long-term patient and graft survival after primary percutaneous coronary intervention for acute myocardial infarction due to saphenous vein graft occlusion. Catheter Cardiovasc Interv. 2005, 65:504-509. 10.1002/ccd.20392 